\title{
Avances y desafíos en cohetería experimental con fines científicos, tecnológicos y militares en la Escuela Militar de Aviación-Fuerza Aérea Colombiana ${ }^{1}$
}

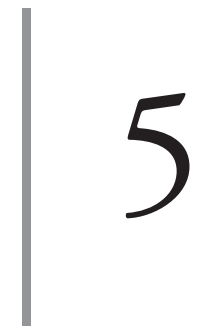

https://doi.org/10.21830/9789585380226.05

\author{
Rafael Andrés Robayo-Salazar \\ Escuela Militar de Aviación "Marco Fidel Suárez"
}

Resumen. El propósito de este capítulo es presentar los avances en cohetería experimental del Grupo de Investigación en Estudios Aeroespaciales (GIEA) de la Escuela Militar de Aviación "Marco Fidel Suárez" (EMAVI). Los avances están relacionados con el desarrollo de componentes de propulsión; el diseño, construcción y caracterización de motores cohete; la síntesis de combustibles sólidos; la obtención y caracterización de materiales compuestos y, recientemente, el diseño y construcción de prototipos de cohetes experimentales aptos para el transporte de cargas útiles tipo CanSat con capacidades de inspección, toma de datos y vigilancia de territorios a bajo costo desde la tropósfera (altura $\leq 10 \mathrm{~km}$ ). Se concluye que estos avances son estratégicos para fomentar el interés hacia la investigación en tecnologías aeroespaciales y cohetería entre los cadetes, oficiales, docentes e investigadores de la EMAVI.

Palabras clave: ciencias del espacio; cohetería experimental; cohetes de sondeo; motores cohete; programa espacial; seguridad y defensa.

1 Este capítulo forma parte de los resultados del proyecto de investigación "FAcson: Desarrollo y construcción de prototipo a escala de cohete sonda con fines científicos, tecnológicos y militares basado en tecnología colombiana”, del Grupo de Investigación en Estudios Aeroespaciales (GIEA), de la Escuela Militar de Aviación "Marco Fidel Suárez" (EmAvi) de la Fuerza Aérea Colombiana, registrado con el código COL0084209 de Minciencias. Los puntos de vista y los resultados presentados en este capítulo pertenecen al autor y no reflejan necesariamente los de las instituciones participantes.

2 Doctor en Ingeniería con énfasis en Ingeniería de los Materiales de la Universidad del Valle. Docente investigador del Programa de Ingeniería Mecánica de la Emavi, Cali, Colombia, y miembro activo del GieA. Reconocido por Minciencias como investigador asociado y par evaluador del Sistema Nacional de Ciencia, Tecnología e Innovación (SNCTEI). ORCID: https:/orcid.org/0000-0003-1687-2885 Contacto: rafael.robayo@emavi.edu.co 


\section{Introducción}

La Fuerza Aérea Colombiana (FAC) se enfrenta constantemente a la necesidad de desarrollar aplicaciones tecnológicas con un alto nivel de novedad en los ámbitos aéreo, espacial militar y de defensa, en cumplimiento de su misión institucional. $\mathrm{Al}$ respecto, proyectar estrategias que le permitan lograr una autonomía y ventaja operacional, desarrollando capacidades propias y dinámicas a través de la investigación en áreas del conocimiento tales como la cohetería experimental, le permitirán a la institución enfrentar las necesidades institucionales y liderar el poder aeroespacial con poca dependencia tecnológica externa, aportando al posicionamiento del país como un referente de este campo a nivel regional.

El desarrollo de proyectos de investigación en cohetería experimental se reconoce como la base para consolidar la escuela de conocimiento e infraestructura necesaria para que países emergentes como Colombia inicien y logren la evolución hacia misiones aeroespaciales en el futuro previsible. La obtención de capacidades que permitan diseñar y construir cohetes de sondeo ofrece ciertas ventajas operacionales en la FAC, debido a que estos vehículos experimentales pueden ser lanzados desde sitios temporales en todo el territorio nacional, pueden utilizarse para estudios de campo remotos, asimismo, se los puede emplear con el fin de desarrollar y poner a prueba nuevos instrumentos científicos (cargas útiles) de medición y observación (Robayo-Salazar, 2019).

Los cohetes experimentales son considerados por la NASA como una herramienta única para llevar a cabo investigaciones científicas en regiones importantes de la atmósfera y/o del espacio que son demasiado bajas para ser muestreadas por satélites (NASA, 2019). En este sentido, los cohetes experimentales son empleados para el transporte, a bajo costo, de instrumentos científicos de medición y observación (cargas útiles), los cuales incluyen un sistema de recuperación. Entre los campos de aplicación se han reportado la biometeorología, fotobiología atmosférica preventiva, monitoreo de contaminación, telecomunicaciones, observación de territorios (seguridad y defensa) y toma de imágenes aéreas, entre otras (Marcado et al., 2009). Además del aporte a la ciencia y la tecnología, las misiones de cohetería experimental también proporcionan herramientas invaluables para la formación académica 
y científica (Acosta et al., 2011), promoviendo, a través de la ejecución de proyectos de investigación, el surgimiento de escuelas de conocimiento necesarias para avanzar hacia misiones más costosas y de mayor duración (Bandecchi et al., 1999), como el lanzamiento de cohetes tipo sonda y la colocación en posiciones suborbitales y orbitales de nano y pico-satélites al servicio del país (Álvarez \& Corredor, 2019).

Con base en lo anterior, la Escuela Militar de Aviación (EMAvi) de la FAC propone el desarrollo de proyectos de investigación relacionados con el diseño, la construcción y el lanzamiento de cohetes de sondeo con fines militares, científicos y experimentales. Estas iniciativas se enmarcan dentro de un macroproyecto de investigación, denominado FACSON, que surgió en la EMAVI con la participación de cadetes, oficiales, docentes e investigadores del Programa de Ingeniería Mecánica (PIMEC) y del Grupo de Investigación en Estudios Aeroespaciales (GIEA). Este macroproyecto de investigación se enmarca dentro de los Programas Estratégicos de Ciencia, Tecnología e Innovación del Modelo de Investigación (MAINV) de la FAC, específicamente el Programa Espacial FAC. En el marco de este programa, el macroproyecto FACSON se relaciona con el subprograma Proyectos de Desarrollo Aeroespacial y sus líneas de investigación: (1) Plataformas y vehículos espaciales, (2) Sistemas de carga espacial y (3) Sistemas de misión espacial. Recientemente, el Pimec de la EMAVI-FAC ha obtenido resultados promisorios en la síntesis de combustibles sólidos (propelentes), diseńo y caracterización de motores cohete, materiales compuestos y diseño de prototipos de cohetes experimentales.

\section{Métodos}

La metodología utilizada para el desarrollo de los proyectos de cohetería experimental en la EMAVI-FAC se enmarca en la ejecución de un macroproyecto denominado FACsON. Este macroproyecto tiene como propósito el diseño, la construcción y el lanzamiento de un prototipo de cohete sonda o experimental. En este sentido, FACSON da origen a diversos microproyectos e investigaciones específicas relacionadas con el desarrollo de cada uno de los componentes y/o subsistemas que componen el cohete. $\mathrm{Al}$ respecto, la figura 1 
resume las fases y/o etapas metodológicas que integran los progresos en cohetería experimental en la EMAVI (macroproyecto FACSON).

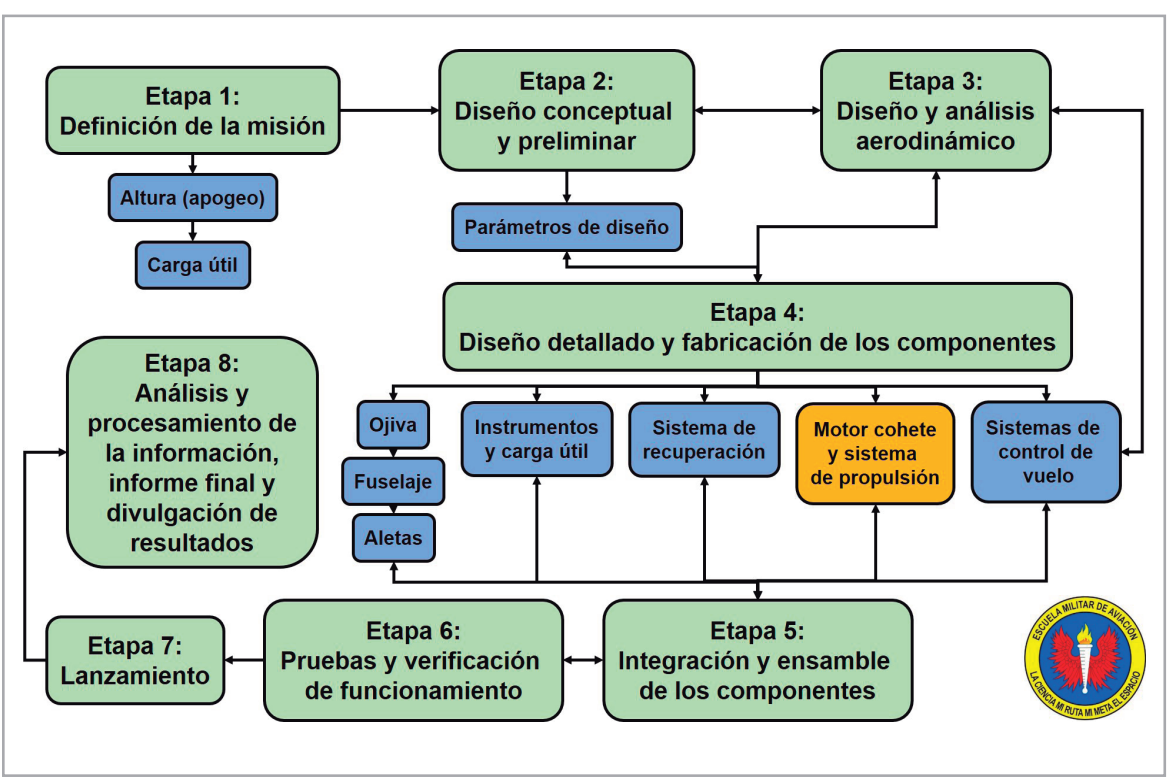

Figura 1. Resumen esquemático de la metodología llevada a cabo en el marco del macroproyecto FaCson desarrollado en la EMAVI-FAC.

Fuente: Elaboración propia.

A la fecha, y como se relaciona en la figura 2, la EMAVI-FAC ha concentrado sus esfuerzos en el diseño, la construcción y la caracterización de motores cohete y sistemas de propulsión, incluyendo: (1) la síntesis del combustible sólido (propelente), (2) el desarrollo de materiales compuestos para la manufactura de componentes de propulsión (cámara de combustión y tobera) y (3) la fabricación y prueba de sistemas de ignición. Complementariamente, se avanza en el diseño y la construcción del fuselaje (ojiva, cuerpo y aletas) de un primer prototipo de cohete, denominado FACSON-0. Algunos de estos avances son incluidos en el presente capítulo (aparte de resultados). Por su parte, se proyecta que los cohetes experimentales permitan el transporte de cargas útiles tipo CanSat, razón por la cual se avanza en su diseño y construcción con el apoyo de Aess Colombia. 


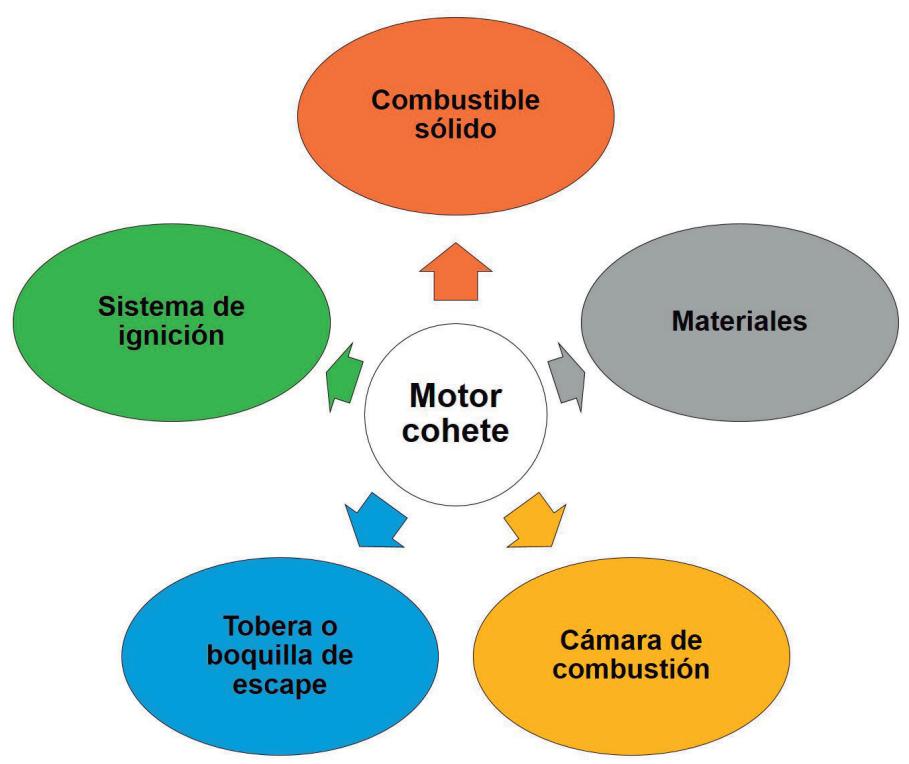

Figura 2. Áreas de investigación en componentes de propulsión y/o motores cohete en la EMAVI-FAC.

Fuente: Elaboración propia.

\section{Resultados y discusión}

El alcance o apogeo de un cohete experimental depende principalmente del desempeño y capacidad de empuje y/o propulsión del motor cohete. Dentro de los tipos de motor cohete comúnmente utilizados se destacan los químicos, los cuales aprovechan la reacción química de distintos compuestos en una cámara de combustión, de forma que se generan gases de alta temperatura y presiones muy altas (Murcia \& Gómez, 2012). Estos gases son entonces acelerados por una tobera de tipo convergente-divergente y son expulsados a gran velocidad para producir el cambio de momentum que impulsa al cohete. Adicional al empuje por momentum, se suma el empuje por presión, que resulta de la diferencia de presiones existentes entre los gases de escape y la atmósfera, en la salida de la tobera. A la mezcla de los compuestos químicos se le llama propelente ('combustible') y a este tipo de motores se les clasifica según el estado físico de este: propelente líquido, propelente sólido, propelente gaseoso y propelente híbrido (Martínez, 2014; Suton \& Biblarz, 2010). 
En cuanto al diseño, construcción y prueba estática de motores cohete en la EMAVI-FAC, los resultados están relacionados con prototipos basados en combustibles (propelentes) sólidos tipo Candy, los cuales han sido utilizados con éxito en el campo de la cohetería experimental (Cubides, 2015; Lacruz et al., 2016). Estos combustibles se basan en la combinación de nitrato de potasio $\left(\mathrm{KNO}_{3}\right)$ (oxidante) y azúcares (combustible). La composición ideal propuesta por Richard Nakka para este tipo de propelentes es de $65 \%$ oxidante y $35 \%$ combustible (en masa) (Nakka, 1997).

Los motores cohete son clasificados de acuerdo con su capacidad de propulsión (impulso específico e impulso total). La tabla 1 relaciona el impulso total (Іт) para las diferentes clases de motores cohete.

Tabla 1. Clasificación de motores cohete según su capacidad de propulsión.

\begin{tabular}{cccccccc}
\hline Clase & \multicolumn{2}{c}{$\begin{array}{c}\text { Impulso total (IT) } \\
\text { (newton-segundos) }\end{array}$} & Clase & \multicolumn{3}{c}{$\begin{array}{c}\text { Impulso total (IT) } \\
\text { (newton-segundos) }\end{array}$} \\
\hline A & 1,26 & - & 2,5 & $\mathrm{~L}$ & 2,560 & - & 5,120 \\
$\mathrm{~B}$ & 2,5 & - & 5 & $\mathrm{M}$ & 5,120 & - & 10,240 \\
$\mathrm{C}$ & 5 & - & 10 & $\mathrm{~N}$ & 10,240 & - & 20,480 \\
$\mathrm{D}$ & 10 & - & 20 & $\mathrm{O}$ & 20,480 & - & 40,960 \\
$\mathrm{E}$ & 20 & - & 40 & $\mathrm{P}$ & 40,960 & - & 81,920 \\
F & 40 & - & 80 & $\mathrm{Q}$ & 81,920 & - & 163,840 \\
G & 80 & - & 160 & $\mathrm{R}$ & 163,840 & - & 327,680 \\
$\mathrm{H}$ & 160 & - & 320 & $\mathrm{~S}$ & 327,680 & - & 655,360 \\
$\mathrm{I}$ & 320 & - & 640 & $\mathrm{~T}$ & 655,360 & - & $1,310,720$ \\
J & 640 & - & 1,280 & $\mathrm{U}$ & $1,310,720$ & - & $2,621,440$ \\
K & 1,280 & - & 2,560 & $\mathrm{~V}$ & $2,621,440$ & - & $5,242,880$ \\
\hline
\end{tabular}

Fuente: Adaptada de Nakka (1997).

Los diseños de los motores en la EMAVI-FAC se han basado en la metodología SRM (Solid Rocket Motor Design). Un primer prototipo (FACSON-0) de motor cohete incluyó el sistema ignitor, la cámara de combustión, el combustible sólido y la tobera (figura 3). La clase del motor seleccionada para fines de diseño se estableció con base en el logro de un impulso total de 80-160 N s (motor clase G). Más adelante retomaremos la caracterización y prueba estática (combustión) de este prototipo de motor cohete. 


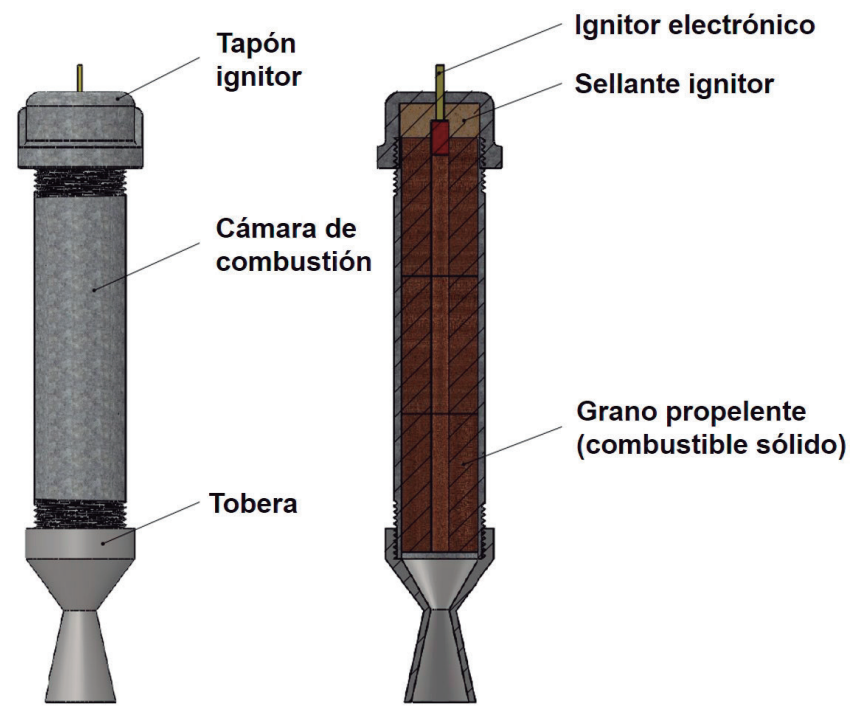

Figura 3. Diseño y construcción de prototipos de motores cohete basados en combustibles sólidos tipo Candy KNSu en la Emavi-Fac.

Fuente: Elaboración propia.

Por su parte, debido a que los componentes del motor cohete deben diseñarse para soportar temperaturas de trabajo superiores a los $1.600-2.000{ }^{\circ} \mathrm{C}$ en un ambiente ablativo severo, la EMAVI-FAC ha desarrollado materiales compuestos de alta temperatura para la fabricación de componentes de propulsión de bajo peso. En efecto, los materiales ablativos se reconocen como materiales fungibles (de sacrificio) cuyas propiedades permiten catalogarlos como sistemas de protección térmica en elementos recuperables que en servicio son expuestos a elevadas temperaturas $\left(\geq 1.500^{\circ} \mathrm{C}\right)$ (NASA, 2008). Las funciones de un material ablativo se resumen en el hecho de ser refractario, aislante térmico, resistente al desgaste, autoconsumible y resistente en cuanto a los esfuerzos térmicos (Maldonado et al., 2014). Generalmente, los materiales ablativos se reconocen por su elevado costo y difícil adquisición, en gran parte debido a los materiales ablativos comúnmente utilizados y la tecnología involucrada en sus procesos de fabricación, lo cual es considerado como una de las principales limitantes para el desarrollo de estas industrias en la región. 
Dentro de los materiales actualmente utilizados para la fabricación de sistemas de protección térmica se destacan los materiales compuestos ablativos de matriz polimérica (CAMP), en gran parte debido a su versatilidad y la posibilidad de reducir el peso $(\approx 90 \%)$ de los componentes de propulsión, sin sacrificar las propiedades ablativas de interés (Astola et al., 2017).

Para el sector aeroespacial y aeronáutico colombiano es una necesidad fabricar CAMP con materiales disponibles localmente, que permitan el impulso de esta industria con poca dependencia tecnológica externa. En este sentido, el Grupo de Investigación en Estudios Aeroespaciales (GIEA) y el Programa de Ingeniería Mecánica (PIMEc) de la EMAVI-FAC han acumulado una experiencia importante relacionada con la obtención y aplicación de CAMP.

Los materiales compuestos desarrollados en la EMAVI (figura 4) han sido caracterizados físico-mecánicamente y sometidos a ensayos ablativos de llama directa $\left(-1.600-2.000{ }^{\circ} \mathrm{C}\right.$ durante 120 segundos). Como resultado se reportan niveles de aislamiento térmico de hasta el 92,9 \% y pérdidas de peso (erosión termomecánica) posteriores al ensayo ablativo inferiores al 13,2\%. Estos resultados han permitido su aplicación en la fabricación de componentes de propul-

a)

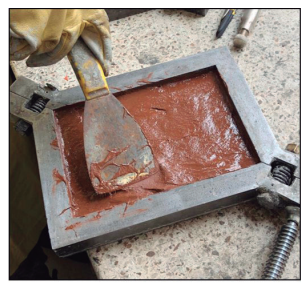

d)

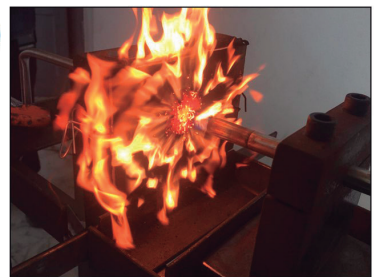

b)

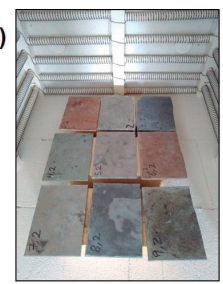

e)

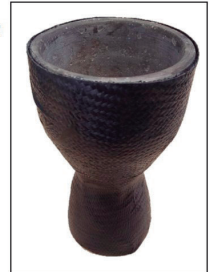

c)

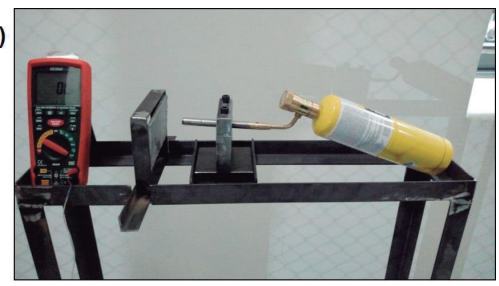

f)

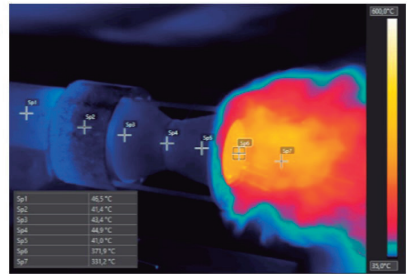

Figura 4. Desarrollo y pruebas térmicas de materiales compuestos ablativos para la fabricación de componentes de propulsión de bajo peso. a. Moldeo de compuestos ablativos; b. Procesamiento de componentes; c. Montaje de pruebas ablativas; d. Comportamiento ablativo de una placa de compuesto; e. Tobera o boquilla de escape fabricada a partir de compuestos ablativos; f. Prueba de combustión real y determinación del perfil térmico alcanzado por la tobera.

Fuente: Elaboración propia. 
sión tipo cámaras de combustión y toberas (boquillas) de motor cohete hasta siete veces más livianas que las convencionales; aplicaciones que se consideran de suma importancia en la búsqueda de disminuir el peso de los vehículos espaciales y aumentar su apogeo en relación con una propulsión específica (Robayo-Salazar et al., 2020). A lo que se le puede sumar el hecho de que los CAMP sintetizados en EMAVI se componen en el $67 \%$ de residuos o subproductos industriales y materiales de fácil adquisición (bajo costo).

Retomando la caracterización de motores cohete y la ejecución de pruebas de combustión estática en la EMAVI, el propelente sólido utilizado para estos ensayos se basó en la mezcla de dos constituyentes principales: un oxidante y un combustible. En el caso de los combustibles sólidos tipo Candy, el oxidante comúnmente utilizado para su síntesis es el nitrato de potasio (KNO3 [KN]). Entre los azúcares (combustible) se destacan: la sucrosa (SU), el sorbitol (SO) y la dextrosa (DX). En efecto, el grano propelente se basó en la mezcla de KN y SU, cuya síntesis se logró a través de un proceso de fusión térmica $(150-170$ C durante 20-25 min) (figura 5).

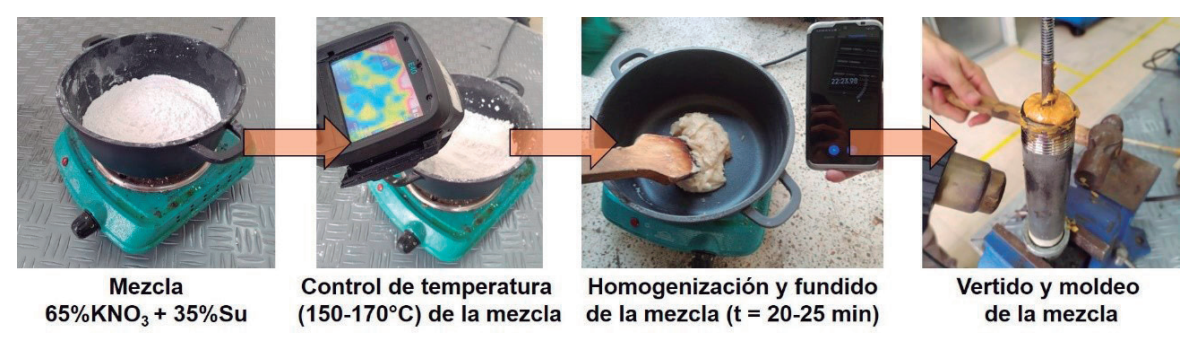

Figura 5. Procesos de síntesis de granos propelentes (combustibles sólidos) en la EMAVI-FAC. Fuente Elaboración propia.

El desempeño del primer prototipo de motor cohete (FACSON-0) fue evaluado mediante una prueba estática de combustión (figura 6a), la cual arrojó como resultados una fuerza de empuje máxima (carga) de $227 \mathrm{~N}$, un impulso total de 98,69 N-s y un impulso específico de 65,8 s. Esto permitió clasificarlo como un motor cohete clase $\mathrm{G}$, en concordancia con los parámetros establecidos inicialmente para su diseño. Complementariamente, se han realizado pruebas de combustión similares con el fin de poner a prueba los componentes 
de propulsión fabricados en materiales compuestos ablativos (CAMP) (figura 6b), mediante las cuales se han obtenido resultados satisfactorios ante condiciones reales de servicio. Una descripción más detallada de dichos hallazgos puede ser consultada en los artículos "Polymeric ablative composite materials and their application in the manufacture of aerospace propulsion components" (Robayo-Salazar et al., 2020) y "Compuestos ablativos de matriz polimérica (resina poliéster) reforzada con materiales de desecho industrial y fibra de vidrio" (Hermann et al., 2019). Los resultados obtenidos son considerados de gran importancia en el desarrollo y la caracterización de motores cohete de combustible sólido, necesarios para impulsar el campo de la cohetería experimental en Colombia.

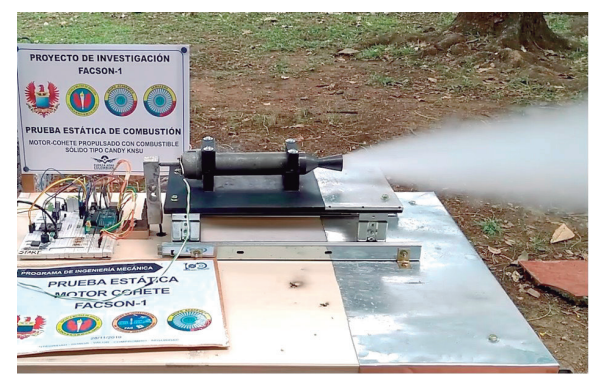

a)

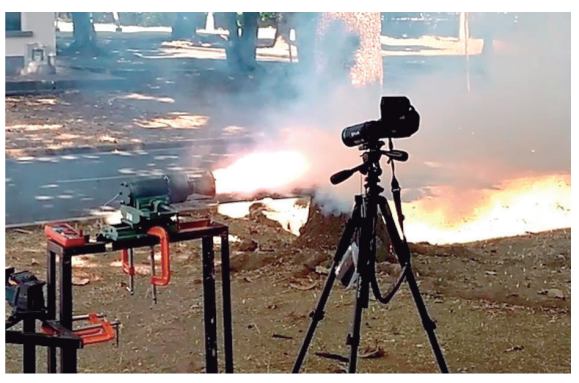

b)

Figura 6. Pruebas de combustión estática para motores cohete en la EMAVI-FAC. a. Determinación del empuje del primer prototipo de motor cohete (FACSON-0); b. Prueba bajo condiciones reales de servicio de los componentes de propulsión (cámara de combustión y tobera) fabricados a partir de materiales compuestos ablativos de bajo peso.

Fuente: Elaboración propia.

Como integración de todos estos desarrollos, la EMAVI-FAC avanza en el diseño de prototipos de cohetes experimentales con el propósito de ser pionero en el lanzamiento de este tipo de vehículos espaciales en la región. En principio, la investigación de cohetes de combustible sólido le permitirá a la institución adquirir toda la escuela de conocimientos necesaria para lograr incursionar en combustibles de mayor capacidad de empuje (mayor apogeo en las misiones experimentales). Los diseños de prototipos de cohetes experimentales en la EMAVI-FAC se han basado en herramientas computacionales de diseño, tales como el software OpenRocket-15. 
Una muestra de la labor investigativa en este campo se representa en la figura 7, la cual corresponde al diseño de un primer prototipo de cohete basado en el motor clase $\mathrm{G}$ reportado anteriormente. Una descripción más detallada de los componentes que integran este prototipo se presenta en la tabla 2. Se espera que este cohete, denominado FACSON-0 (primer prototipo del macroproyecto FACSON), sea lanzado en el año 2021. Especialmente, se debe destacar que sería un cohete $100 \%$ diseñado y fabricado en la institución con la participación de cadetes, profesores, investigadores y oficiales de la EMAVI.
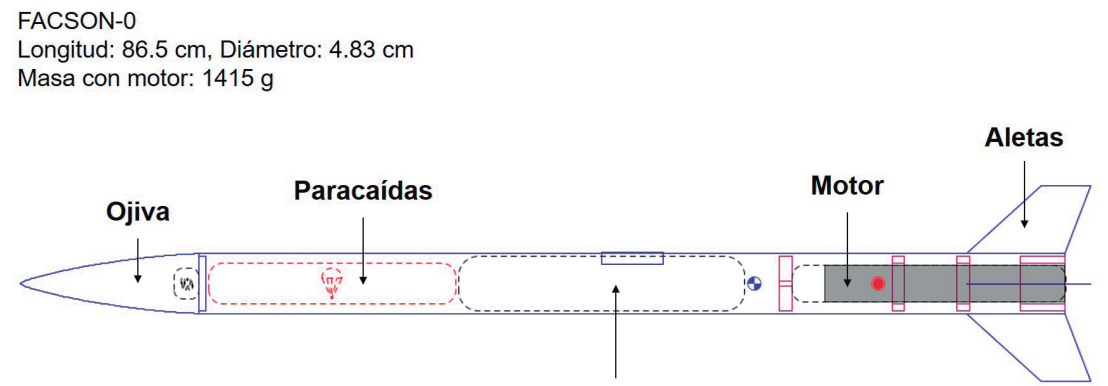

Apogeo: $462 \mathrm{~m}$

Carga útil

Velocidad máx.: $89.6 \mathrm{~m} / \mathrm{s}$

Aceleración máx.: $47.7 \mathrm{~m} / \mathrm{s}^{2}$

Estabilidad: $2.07 \mathrm{cal}$

CG: $59.4 \mathrm{~cm}, \mathrm{CP}: 69.4 \mathrm{~cm}$

Figura 7. Diseño preliminar del cohete experimental Facson-0 de la EMavi-Fac. Datos importados del Software OpenRocket versión 15.03.

Fuente: Elaboración propia.

Tabla 2. Detalles de los componentes que conforman el Facson-0. Datos importados del Software OpenRocket versión 15.03

\begin{tabular}{|c|c|c|c|c|c|}
\hline$<$ & Ojiva & $\begin{array}{c}\text { ABS } \\
(1,02 \mathrm{~g} / \mathrm{cm} 3)\end{array}$ & Series Haack & $\begin{array}{l}\text { Len: } \\
14,5 \mathrm{~cm}\end{array}$ & $\begin{array}{l}\text { Mass: } \\
28,9 \mathrm{~g}\end{array}$ \\
\hline 0 & Cuerpo & $\begin{array}{c}\text { PVC } \\
(1,39 \mathrm{~g} / \mathrm{cm} 3)\end{array}$ & $\begin{array}{l}\text { Dia(in) } 4,36 \mathrm{~cm} \\
\operatorname{Dia}(\text { out } 4,83 \mathrm{~cm}\end{array}$ & $\begin{array}{l}\text { Len: } \\
70 \mathrm{~cm}\end{array}$ & $\begin{array}{l}\text { Mass: } \\
330 \mathrm{~g}\end{array}$ \\
\hline & $\begin{array}{c}\text { Aletas } \\
\text { tapezoidales } \\
(4)\end{array}$ & $\begin{array}{c}\text { PVC } \\
(1,39 \mathrm{~g} / \mathrm{cm} 3)\end{array}$ & Thick: $0,2 \mathrm{~cm}$ & & $\begin{array}{l}\text { Mass: } \\
41,5 \mathrm{~g}\end{array}$ \\
\hline
\end{tabular}




$\begin{array}{ccccc}\text { Masa } & \text { Dia(out) } 4,42 \mathrm{~cm} & \text { Mass: } \\ 250 \mathrm{~g}\end{array}$

Fuente: Elaboración propia.

Los avances obtenidos hasta ahora en la EMAVI-FAC, relacionados con la cohetería experimental, se reconocen como un primer paso, enfocado especialmente en la generación de una cultura investigativa e interés en los cadetes (futuros oficiales de la FAC) hacia los temas aeroespaciales. Sin embargo, algunos retos de gran escala han sido identificados y se describen a continuación como oportunidades para lograr el posicionamiento de las tecnologías aeroespaciales en el país y finalmente aportar a la seguridad y la defensa de la región.

\section{Conclusiones}

Los resultados obtenidos en la EMAVI-FAC, relacionados con (1) el diseño y la caracterización de motores cohete, (2) la síntesis de combustibles sólidos 
(propelentes), (3) la fabricación de componentes de propulsión basados en materiales compuestos de bajo peso, y (4) el diseño de prototipos de cohetes experimentales, son promisorios y han fomentado la formulación y ejecución de diversos proyectos de investigación y trabajos de grado, que, a su vez, son complementarios entre sí y se enmarcan dentro del macroproyecto denominado Facson. Cabe destacar que estos proyectos involucran un número importante de cadetes, oficiales, docentes e investigadores del Grupo de Investigación en Estudios Aeroespaciales (GIEA) y el Programa de Ingeniería Mecánica de la Escuela Militar de Aviación (EMAvi), los cuales concatenan esfuerzos con investigadores y profesionales de otras instituciones a nivel regional y nacional para generar una cultura investigativa alrededor del campo de la cohetería experimental. La articulación de todos estos actores y el fortalecimiento de sus labores investigativas se consideran un motor para impulsar, desde la academia, el sector aeroespacial del país, aportando a la seguridad y la defensa de la región.

\section{Referencias}

Acosta Vásquez, E. A., Marín, L. E., \& Jaimes, O. (2011). Cohetes hidráulicos: Diseño, construcción y experimentación, una experiencia integradora. Revista Científica, 13(1), 229-231. https://doi.org/10.14483/23448350.966

Álvarez Calderón, C. E., \& Corredor Gutiérrez, C. G. (2019). El espacio exterior: Una oportunidad infinita para Colombia. Fuerza Aérea Colombiana.

Astola, P. J., Rodríguez, M. A., Botana, F. J., \& González-Rovira, L. (2017). Caracterización de elementos de protección térmica de materiales compuestos mediante análisis térmicos. Revista de la Asociación Española de Materiales Compuestos, 2(4), 34-41.

Bandecchi, M., Melton, B., \& Ongaro, F. (1999). Concurrent engineering applied to space mission assessment and design. Esa Bulletineuropean Space Agency, (99), 34-40.

Cubides, C. (2015). Propelentes sólidos: una experimentación para el futuro. Revista Ciencia y Tecnología del Ejército, 6(12), 11-19.

Hermann, J. P., Osorio, J. D., \& Robayo-Salazar, R. A. (2019). Compuestos ablativos de matriz polimérica (resina poliéster) reforzada con materiales de desecho industrial y fibra de vidrio. Revista Ingenierías Universidad de Medellin, 19(36), 223-237. https://doi. org/10.22395/rium.v19n36a11

Lacruz, L., Parco, M., Santos, R., Torres, C., Ferreira, J., \& Benítez-Díaz, P. (2016). Análisis experimental de las oscilaciones de presión interna en un motor de combustible sólido para cohete sonda. Revista Ciencia e Ingeniería, 37(2), 81-88. 
Maldonado Villa, J., Portocarrero Hermann, J., Rodríguez Adaime, C., Valbuena Cocunubo, J. J., \& Acuña Lizarazo, M. E. (2014). Evaluación del comportamiento térmico de materiales compuestos de matriz polimérica en prototipos de toberas para cohetes de órbita baja. Revista Cientifica General José María Córdova, 12(13), 275-290. https://doi. org/10.21830/19006586.163

Marcado, V., Benítez, P., La Rosa, C., La Cruz, L., Parco, M., Ferreira, J., Andressen, R., Serra Valls, A., Peñaloza, M., Rodríguez, L., Cárdenas, J. E., Minitti, V., \& Rojas, J. J. (2009). Progresos alcanzados en el proyecto universitario cohete sonda ULA. Universidad, Ciencia y Tecnología, 13(53), 305-316. https://doi.org/10.13140/RG.2.1.3286.5120

Martínez Pino, D. (2014). Diseño y optimización de un cohete de sondeo. Universidad de Sevilla.

Murcia Piñeros, J., \& Gómez Salcedo, S. (2012). Diseño de los motores cohetes de propelente sólido para el despegue asistido de un vehículo aéreo no tripulado (VANT). Ciencia y Tecnología Aeronáutica, (17), 29-39.

Nakka, R. (1997). Richard Nakka’s Experimental Rocketry Web Site. http://www.nakka-rocketry.net/

NASA. (2008). Sistema y materiales de protección térmica (TPS). https://www.nasa.gov/centers/ ames/spanish/research/humaninspace/humansinspace-thermalprotec.html

NASA. (2019). Descripción general del programa Rocket de sondeo de la NASA: Oportunidades únicas para investigación científica enfocada, de bajo costo y rápida. https://rscience.gsfc. nasa.gov/srrov.html

Robayo-Salazar, R. A. (2019). Cohetes tipo sonda con fines científicos, tecnológicos y militares: una oportunidad para su desarrollo en la EMAVI-FAC. En Ciencia, gestión e ingeniería en el desarrollo aeroespacial (pp. 49-72). Escuela Militar de Aviación "Marco Fidel Suárez".

Robayo-Salazar, R. A, Portocarrero-Hermann, J., Díaz-Padrón, U., \& Patiño-Castrillón, O. (2020). Polymeric ablative composite materials and their application in the manufacture of aerospace propulsion components. Revista Facultad de Ingeniería, 29(54), e10662. https://doi.org/10.19053/01211129.v29.n54.2020.10662

Suton, G. P., \& Biblarz, O. (2010). Rocket propulsion elements (5.a ed.). John Wiley \& Sons. 